\title{
Acai Berry Induced Cholestatic Jaundice
}

\author{
Leanne Stratton a, Joanne McNeilly ${ }^{\mathrm{a}}$, Ciaran O’Neill' \\ William Jonathan Cash ${ }^{\mathrm{a}, \mathrm{c}}$
}

\begin{abstract}
A previously well 52-year-old gentleman presented with a 2-week history of itch, jaundice, lethargy, pale stools and dark urine. He had no risk factors for liver disease. On systematic questioning, the only new addition to his diet was an acai berry supplement drink to promote weight loss. Blood tests revealed markedly raised bilirubin and ALP, and slightly raised AST and ALT. Ferritin was also raised but was felt to represent an acute phase reactant. The remaining liver screen was unremarkable. Imaging showed a $12 \mathrm{~mm}$ hemangioma in segment VII of the liver but was otherwise normal, with no ductal dilatation noted. ERCP revealed normal ducts. Liver biopsy was performed and this revealed hepatocellular and canalicular cholestasis with minimal parenchymal inflammation. Portal tracts were inflamed with lymphocytes. Findings were felt to be consistent with ductal obstruction or drug reaction. Overall findings were felt to be in keeping with a drug reaction secondary to the acai berry supplement. He had been commenced on prednisolone, ursodeoxycholic acid, cholestyramine, lansoprazole and chlorphenamine in a district general hospital prior to transfer to the Liver Unit. On arrival, naltrexone was added to the regimen. His bilirubin peaked at $284 \mu \mathrm{mol} / \mathrm{L}$ but soon decreased, and returned to normal levels within 2 months. High levels of D-aspartate are found in these berries, and this amino acid is involved in a pituitary pathway involved in stimulation of testosterone production. Alongside anabolic steroids, androgenic steroids have also been shown to be involved in development of cholestatic jaundice. Beta-sitosterol is also found in the berries and is a precursor of boldenone which is an anabolic steroid. These steroid pathways are among the hypotheses for development of cholestatic jaundice in this gentleman.
\end{abstract}

Keywords: Cholestasis; Acai berry; Drug induced liver injury; Jaundice

\footnotetext{
Manuscript accepted for publication May 6, 2014

${ }^{a}$ Liver Unit, Royal Victoria Hospital, Belfast, UK

${ }^{b}$ Department of Cellular Pathology, Antrim Area Hospital, Antrim, UK

${ }^{\mathrm{c}}$ Corresponding Author: WJ Cash, Liver Unit, Royal Victoria Hospital,

Belfast BT12 6BA, UK. Email: Johnny.cash@belfasttrust.hscni.net
}

doi: http://dx.doi.org/10.14740/jmc1794w

\section{Introduction}

Drug induced liver injury is a potential diagnosis that should be considered in all patients presenting with newly deranged liver enzymes. This is particularly the case when patients have no clear risk factors for developing liver disease, and when there has been a rapid onset of symptoms and deterioration in function. Alongside paracetamol which should always be considered, other more insidious drugs may bring about hepatotoxicity, including antibiotics and antiepileptic medication. Increasingly patients present having taken herbal remedies of unknown constituency. This is worrying as the exact components and their effects are unknown. This report focuses on one such case, whereby the offending agent was thought to be an acai berry supplement.

\section{Case Report}

A previously well 52-year-old male presented with a 2-week history of itch, jaundice, pale stools, dark urine and general malaise. He did not have any significant past medical history. He did not take regular prescribed medication, nor had he taken antibiotics within the preceding months. He had, however, been taking an acai berry supplement drink on a daily basis to facilitate weight loss for a few months. There was no family history of liver disease. He was a non-smoking businessman and consumed approximately 12 units of alcohol per week. There were no risk factors for viral hepatitis such as intravenous drug abuse, tattoos, blood transfusions or recent travel.

Initial liver function tests (LFTs) demonstrated a mixed but predominantly cholestatic pattern, with a bilirubin of 90 $\mu \mathrm{mol} / \mathrm{L}(4-17 \mu \mathrm{mol} / \mathrm{L})$ on admission to the Liver Unit. The patient became progressively more jaundiced and bilirubin peaked at $284 \mu \mathrm{mol} / \mathrm{L}$ with associated enzymes of ALP 215 U/L (35 - $104 \mathrm{U} / \mathrm{L})$, AST $51 \mathrm{U} / \mathrm{L}$ (5 - $40 \mathrm{U} / \mathrm{L})$, GGT $34 \mathrm{U} / \mathrm{L}$ (11 - $50 \mathrm{U} / \mathrm{L})$, ALT $75 \mathrm{U} / \mathrm{L}(5-40 \mathrm{U} / \mathrm{L})$ and albumin $39 \mathrm{~g} / \mathrm{L}$ $(35-55 \mathrm{~g} / \mathrm{L})$. His coagulation screen was normal throughout, as was his renal function and full blood picture. Serology for hepatitis A, B and C was negative, as was serology for 


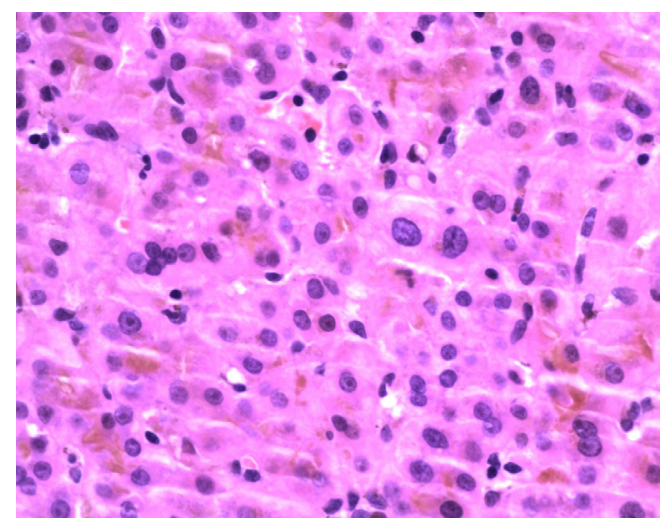

Figure 1. Presence of neutrophil component $(\times 400)$. This, alongside cholestasis, is suggestive of large bile duct obstruction.

CMV, EBV and HSV. His anti-smooth muscle, anti-nuclear and anti-mitochondrial autoantibodies were negative. His IgG level was normal at $13.4 \mathrm{~g} / \mathrm{L}, \mathrm{IgG} 40.75 \mathrm{~g} / \mathrm{L}$ (normal < $1.3 \mathrm{~g} / \mathrm{L}$ ). His ferritin was elevated at 1,622, interpreted as an acute phase reactant, as his $\mathrm{Hb}$ and his iron saturation levels were normal at $129 \mathrm{~g} / \mathrm{L}$ and $37.5 \%$ respectively. Leptospirosis and Q fever IgM antibody tests were negative. Serum copper and ceruloplasmin levels were normal. His ACE level was normal at $36 \mathrm{U} / \mathrm{L}$. Alpha 1-antitrypsin level was normal.

Ultrasound of his abdomen, computerized tomography scan of chest/abdomen/pelvis and magnetic resonance cholangio-pancreatography revealed only a $12 \mathrm{~mm}$ hemangioma in segment VII of the liver. There was no evidence of ductal dilatation and no gallbladder calculi were seen. The pancreas was normal.

Liver biopsy (Fig. 1, 2) revealed hepatocellular and canalicular cholestasis with minimal parenchymal inflammation. All the portal tracts were inflamed with lymphocytes, some plasma cells, some histiocytes, a number of neutrophils but inconspicuous numbers of eosinophils. The morphology did not suggest PSC or PBC and histological findings were felt to be due to a drug reaction or ductal obstruction. ERCP revealed normal ducts.

The only new non-food substance the patient had consumed in the previous 12 months was an acai berry supplement and this was felt to be the most likely cause of the drug induced jaundice.

Ursodeoxycholic acid, cholestyramine, chlorphenamine, prednisolone and lansoprazole had been commenced in a district general hospital prior to transfer to the Liver Unit. Naltrexone was added on arrival to the Unit. His jaundice began to settle and he was discharged when his bilirubin was half of its peak level on a reducing course of prednisolone. Early review in clinic showed marked reduction in cholestatic LFTs, and bilirubin had returned to $10 \mu \mathrm{mol} / \mathrm{L}$ less than 2 months after his admission. He remains well over a year later.

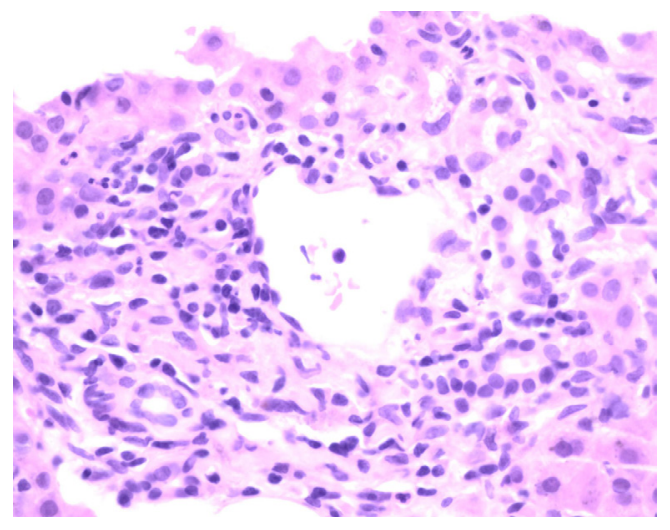

Figure 2. Portal tract demonstrating occasional neutrophils but predominantly lymphocytes and macrophages ( $\times 400)$. Note eosinophils are conspicuously absent.

\section{Discussion}

The acai berry (Euterpe oleracea Mart.) has gained popularity in recent years as a "superfood" due to its high levels of superoxides [1]. From this, it has been assumed that it rivals other highly anti-oxidant foods, although little is currently known about its risks and benefits. No study has as yet proven the health benefits of this berry $[2,3]$.

In Central and Southern America, where the acai palm originates, this small purple berry is regarded as having a number of potent health benefits. Historically these have been regarded as improving virility, treating fevers, aiding parasitic infections and increasing energy levels [4]. Recently, they have been marketed as a therapeutic aid to cancer, arthritis, high cholesterol, erectile dysfunction and weight loss [5]. Indeed it is for this reason that the berry has become so widely used in recent years, and indeed why our subject had been using this supplement.

Anthocyanins contained in the fruit are generally considered to be the major contributors to the antioxidant activity of the berry [6]. Oxidative stress, which is an imbalance between the generation of free radical species and the activity of anti-oxidant defence mechanisms, is thought to be one of the underlying mechanisms behind obesity. Subjects who are obese are more likely to have higher levels of oxidative stress than those of normal weight [7]. Hence acai berries, with their anti-oxidant properties, have been promoted for weight loss, although as yet there is no absolute proof of their efficacy for such an indication. It is also for this anti-oxidant property that they are marketed as anti-atherosclerotic, although similarly there is no study to date proving that this is the case [8].

With regards to liver disease, one study using rats has examined the use of the acai berries as a protective factor against fatty liver disease [9]. This study compared highcholesterol diets in rats, with those on the same diet but also given acai berries. When the livers were removed and com- 
pared, the non-berry group was shown to have heavier livers, presumed to be due to worsened fatty change. However on histology, there was no significant difference between the two groups, and so no proven benefit to liver function was found.

Another hypothesis with regards to their health benefits involves use in chronic disease states. Flavonoids are found in high concentration in these berries, and these have been shown to enhance the number of inhibitory cytokines in circulation, for example of interleukin-6 and TNF-alpha [10]. It is for this reason that they are considered to have antiinflammatory action, and also that they may reduce the risk of neoplastic disease, but as yet this remains unproven.

One theory as to why this gentleman developed such profound jaundice lies in one of the historically noted benefits of this berry - that it enhances virility. Two different mechanisms may provide an explanation for this. Firstly, one of the main amino acids, D-aspartate, has been found in high quantities in this food stuff. This amino acid is found in high concentrations in both the pituitary gland and in the testes. It has been shown to stimulate the production and release of luteinising hormone from the pituitary, and in turn stimulates the release of testosterone [11]. One of the main drugs that should be considered in such cholestatic jaundice, is anabolic steroids. However androgenic steroids have also been shown to have this effect. Testosterone is a naturally occurring steroid, but it may be that the high concentration of D-aspartate in the acai berries stimulated increased release of luteinising hormone and thus testosterone, and that this extra testosterone induced cholestasis in this gentleman. Unfortunately there is no record of testosterone level in this gentleman at any point during his admission.

A similar theory can be proposed as to the chemical constituents of these berries. In every gram, $0.44 \mathrm{mg}$ of beta-sitosterol is found [4]. This is a phytosterol, or steroid found in plants. This chemical is the pre-cursor of boldenone, which is an anabolic steroid. As with the increased production of testosterone, it may be that this gentleman's liver reacted to the increased level of anabolic steroid in circulation, and as such, cholestasis developed.

\section{Conclusion}

We propose that the acai berry was the most likely cause of this gentleman's presentation, and on withdrawal of the supplement, the disease regressed. Although the exact mechanism is unclear, it is possible that there were increased levels of circulating steroids post ingestion of these berries resulting in the cholestatic LFTs that were present on admission. More research is required into the health benefits and risks associated with these berries before their promotion as a "superfood".

\section{Conflict of Interest}

No conflicts of interest to declare.

\section{References}

1. Schauss AG, Clewell A, Balogh L, Szakonyi IP, Financsek I, Horvath J, Thuroczy J, et al. Safety evaluation of an acai-fortified fruit and berry functional juice beverage (MonaVie Active ${ }^{\circledR}$ ). Toxicology. 2010;278(1):46-54.

2. Marcason $\mathrm{W}$. What is the acai berry and are there health benefits? J Am Diet Assoc. 2009;109(11):1968.

3. National Center for Complementary and Alternative Medicine at the National Institutes of Health.Acai. NCCAM Publication; April 2011. Available from: http://nccam.nih.gov/health/acai/ataglance.htm.

4. Heinrich M, Dhanji T, Casselman I. Acai (Euterpe oleracea Mart.) - A phytochemical and pharmacological assessment of the species' health claims. Phytochemistry Letters. 2011;4:10-21.

5. Zeratsky K, Mayo Clinic. Acai berry products: Do they have health benefits? Mayo Foundation for Medical Education and Research; 2010 May 22. Available from: http://www.mayoclinic.com/health/acai/AN01836.

6. Udani JK, Singh BB, Singh VJ, Barrett ML. Effects of Acai (Euterpe oleracea Mart.) berry preparation on metabolic parameters in a healthy overweight population: a pilot study. Nutr J. 2011;10:45.

7. Grattagliano I, Palmieri VO, Portincasa P, Moschetta A, Palasciano G. Oxidative stress-induced risk factors associated with the metabolic syndrome: a unifying hypothesis. J Nutr Biochem. 2008;19(8):491-504.

8. Xie C, Kang J, Burris R, Ferguson ME, Schauss AG, Nagarajan $\mathrm{S}, \mathrm{Wu} \mathrm{X}$. Acai juice attenuates atherosclerosis in ApoE deficient mice through antioxidant and anti-inflammatory activities. Atherosclerosis. 2011;216(2):327-333.

9. de Souza MO, Silva M, Silva ME, Oliveira Rde P, Pedrosa ML. Diet supplementation with acai (Euterpe oleracea Mart.) pulp improves biomarkers of oxidative stress and the serum lipid profile in rats. Nutrition. 2010;26(7-8):804-810.

10. Xie C, Kang J, Li Z, Schauss AG, Badger TM, Nagarajan $\mathrm{S}, \mathrm{Wu} \mathrm{T}$, et al. The acai flavonoid velutin is a potent anti-inflammatory agent: blockade of LPS-mediated TNF-alpha and IL-6 production through inhibiting NFkappaB activation and MAPK pathway. J Nutr Biochem. 2012;23(9):1184-1191.

11. Topo E, Soricelli A, D'Aniello A, Ronsini S, D'Aniello $\mathrm{G}$. The role and molecular mechanism of D-aspartic acid in the release and synthesis of LH and testosterone in humans and rats. Reprod Biol Endocrinol. 2009;7:120. 